\title{
Augmentation of Class-E PA reliability under load mismatch conditions
}

\author{
Jeroen Ponte, Ali Ghahremani, Maikel Huiskamp, Anne-Johan Annema and Bram Nauta \\ dept. of Electrical Engineering, Mathematics and Computer Science \\ University of Twente, Enschede, The Netherlands.
}

j.ponte@ student.utwente.nl

\begin{abstract}
Highly efficient switch-mode Class-E power amplifiers (PAs) are sensitive to load impedance variations. For voltage standing wave ratios (VSWRs) up to 10:1, the peak switch voltage and average switch current can increase by a factor 1.7 and 2.5 , respectively, with respect to the nominal load condition, thereby imposing serious reliability risks. This work introduces a technique to self-protect/self-heal Class-E PAs against the effects of load variations, with only a minor impact on output power and efficiency. To validate the proposed technique, load-pull measurements are conducted on a ClassE PA implemented in a standard 65nm CMOS technology, employing an off-chip matching network, augmented with a fully automated self-protective/self-healing control loop. Under nominal operating conditions, the PA provides $17.5 \mathrm{dBm}$ output power into $50 \Omega$ from a $1.2 \mathrm{~V}$ supply with $67 \%$ efficiency when all the losses of the matching network are included. It is shown that the proposed self-protective PA can reduce its peak switch voltage to below the value allowed by the technology for all load mismatch conditions with VSWR up to 19:1 while output power and efficiency are not considerably affected. Furthermore, a PA with an average current safety factor of 2.5 can reliably handle VSWRs up to 19:1.
\end{abstract}

Index Terms-Load mismatch, VSWR, power amplifiers (PAs), Class-E PA, self-healing.

\section{INTRODUCTION}

$\mathrm{P}$ OWER amplifiers (PAs) are responsible for a sizable portion of power consumption in RF systems, making PA efficiency an important parameter [1]. For this reason, switchedmode PAs (SMPAs) with their high efficiency are an attractive option. Class-E SMPAs employ resonant networks to shape their waveforms to achieve e.g. zero-voltage switching (ZVS) and/or zero-slope switching (ZSS) conditions to ensure high efficiency (ideally 100\%) [2].

Antenna impedances can vary significantly due to changing antenna environment, with VSWRs as high as 10:1 [3]. Nonnominal load conditions detune the SMPA's resonant networks, in some cases leading to degraded output power and efficiency levels. Additionally, extreme switch voltage and current waveforms can result, jeopardizing PA reliability. Switch voltages exceeding the breakdown level may cause instantaneous and permanent failure through gate-oxide break down [4], while high switch currents can cause gradual deterioration or destruction through electro-migration [5] and hot carrier degradation [6]. The load-pull contours in our work in [7] already demonstrated the effect of load mismatch on the switch voltage of a Class-E PA.

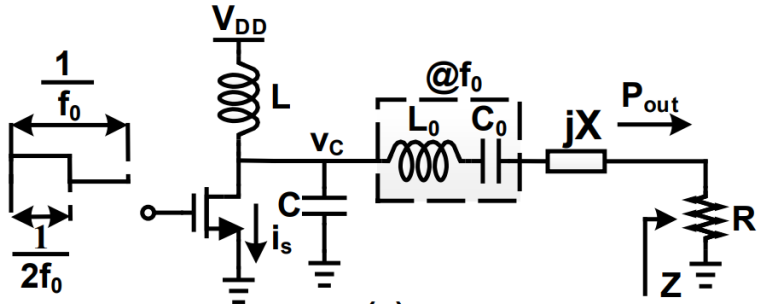

(a)
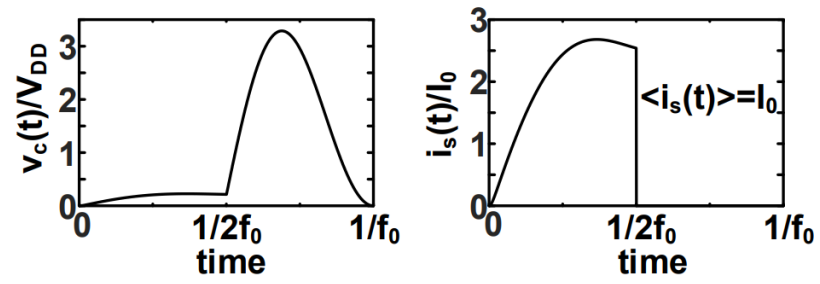

(b)

Fig. 1: (a) Schematic representation of a single-ended Class-E PA. (b) Switch voltage and current waveforms for nominal load, $\mathrm{m}=0.05$, ZVS and ZSS

There are publications on protecting PAs under non-nominal load conditions. In [8], a tunable matching network is used with a linear Class-A PA to enable self-healing for high performance under load mismatch. This technique can also be used for protection against load-mismatch, at the cost of reduced efficiency and increased complexity due to need for a tunable network. Another technique, also for linear PAs, involves placing a feedback loop around the PA to detect peak output voltage and use this to tune the bias current of the PA, to ensure both linearity and safe operation of the PA [9]. Voltage/current limiters were also reported for protection [10], [11], at the cost of compromising both PA functionality and efficiency due to e.g. shunting high currents to ground to limit transistor drain voltage. Lastly, isolators/circulators may be used to isolate the PA from mismatches at the load. However, their dissipative operation makes them unsuitable for use in high-efficiency PA systems.

For Class-E PAs, to the best of the authors' knowledge, there is only one work addressing protection against high VSWRs [12]. The work in [12], similar to that on linear PAs, employs a feedback loop to detect high peak voltage at the drain of the switch and reduces the drive power to limit the switch voltage. However, this compromises switch-mode operation (and hence efficiency) because the drive power must be reduced below the 
level where it adequately switches the switch transistor.

In this work, we propose a new technique to improve the reliability of Class-E PAs under heavy load mismatch. The technique is based on the rotation of the load-pull contours and hence the safe operating region on the Smith chart, introduced in [7]. Furthermore, it is shown that PA efficiency is hardly degraded. Finally, a fully automated self-protective Class-E PA demonstrator is used for experimental verification.

In section II the general principles and behavior under load mismatch of Class-E PAs are treated. Section III describes our demonstrator implementation and shows measurements on this self-healing system. Section IV summarizes the conclusions.

\section{CLASS-E PAS UNDER (NON-)NOMINAL CONDITIONS}

Single-ended Class-E PAs, in their simplified form, consist of a switch (transistor) and two LC tanks. A schematic representation of a single-ended Class-E PA can be found in Fig. 1. The first tank, $L-C$, is used for waveform shaping and the second tank, $L_{0}-C_{0}$, is a band-pass filter tuned to the input frequency, $f_{0}$, to ensure a sinusoidal load current. In the ideal case, the switch is driven by a $50 \%$ duty cycle square wave with frequency $f_{0}$.

For Class-E PAs designed for ZVS and ZSS conditions, parameters $\alpha$ and $\beta$, which represent the level of violation of ZVS and ZSS conditions, are zero; $\alpha$ and $\beta$ are defined as [6]

$$
v_{c}\left(\frac{1}{f_{0}}\right)=\alpha V_{D D}, \quad \frac{d v_{c}}{d t}\left(\frac{1}{f_{0}}\right)=2 \pi \beta f_{0} V_{D D}
$$

where $v_{c}$ is the switch voltage and $V_{D D}$ is the supply voltage. The relations between the Class-E PA parameters and circuit components $L, C, X$ and $R$ are formulated in e.g. [6], in the so-called K-design set $K=\left\{K_{L}, K_{C}, K_{X}, K_{P}\right\}=$ $\left\{\frac{2 \pi L f_{0}}{R}, 2 \pi f_{0} R C, \frac{X}{R}, \frac{R P_{o u t}}{V_{D D}^{2}}\right\}$.

The K-design set parameters depend only, see [6], on $\alpha$, $\beta$, the relative resonance frequency of the LC tanks, $q=$ $1 / 2 \pi f_{0} \sqrt{L C}$, and the technology dependent relative switch on-resistance, $m=2 \pi f_{0} R_{o n} C$. The only dependencies of $m$ are on the technology and the operation frequency [6].

For a Class-E PA which is designed for a certain $q$ and ZVS and ZSS conditions, load-pull contours can be derived, similar to [7]. The resultant contours show the impact of changing load conditions on PA output power, efficiency and (reliability related) maximum switch voltage and average switch current. To achieve this, the PA load, $Z$ with its nominal value, $R$, corresponding to the center of the Smith charts in Fig. 2, is changed and switch voltage and currents can be found using basic circuit theory. While a full derivation is beyond the scope of this manuscript, the results are used to plot output power and average switch current normalized to where $Z=R(\mathrm{VSWR}=1: 1)$, efficiency, and maximum switch voltage normalized to $V_{D D}$. These results are illustrated in Fig. 2 .

For high output power, we take $q \approx 1.4$ [6], while the parameter $m$ for standard $65 \mathrm{~nm}$ CMOS technology in the low $\mathrm{GHz}$ range is about $m \approx 0.05$. To represent the behavior of the Class-E PA for a wide range of load conditions, the use

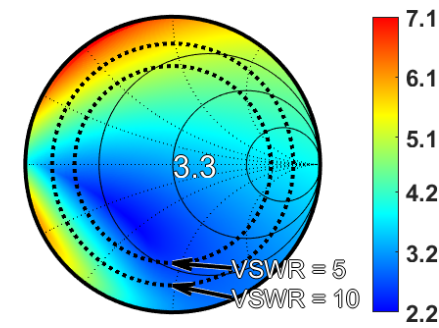

(a) Normalized peak switch

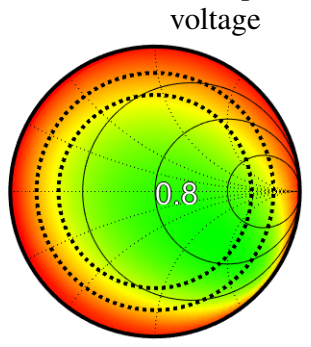

(c) Efficiency

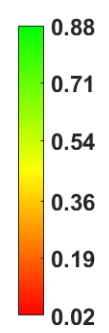

.02

(c) Efficiency

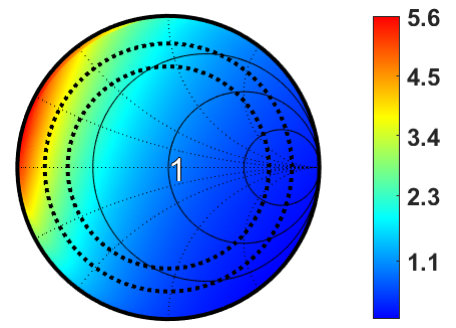

(b) Normalized average switch

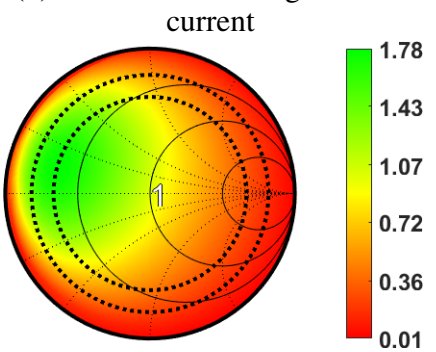

(d) Normalized output power
Fig. 2: Theoretical Class-E PA load-pull color gradients

of parameter color gradients on a Smith chart is employed. Constant VSWR circles for VSWR $=\{10,5\}$ are also shown.

The contours show that the PA displays peak switch voltages and average switch currents below those for the nominal case for a large portion of the Smith chart, with significantly higher switch voltages and currents mainly occurring in the upper left. Both output power and PA efficiency are heavily affected, reaching near-zero values at their individual extremes. Of the shown effects, breakdown voltage-exceeding switch voltage excursions present the greatest risk to PA reliability, rapidly causing permanent device breakdown and these are therefore the primary focus of the remainder of this paper.

\section{A. Effects of the q parameter on PA behavior}

In [7] it was shown that reducing (increasing) $q$ rotates the PA contours counterclockwise (clockwise). Since the reliability of the non-tuned PA is compromised mainly in the upper left of the Smith chart, there we reduce $q$ to rotate the contours in the counterclockwise direction. Resultingly, the safe operating area then includes this previously high risk area on the Smith chart. Decreasing $q$ requires that the capacitor $\mathrm{C}$ be controllable to a value larger than the nominal case.

\section{IMPLEMENTATION}

To decrease PA sensitivity to load mismatch, the $q$ parameter can be tuned by varying its switch capacitance $C$ when mismatch occurs. A schematic representation of an implementation of the principle, using a switch capacitor bank (SCB), is shown in Fig. 3(a).

To demonstrate the tuning and self-healing principles, measurements were performed on a system reusing the $65 \mathrm{~nm}$ CMOS PA chip described in [7]. The switch consists of a $1.2 \mathrm{~V}$ thin oxide transistor cascoded by a thick oxide $2.5 \mathrm{~V}$ transistor with its gate biased to $1.8 \mathrm{~V}$, allowing a maximum switch voltage of up to $4.6 \mathrm{~V}(1.8 \mathrm{~V}+1.1 \times 2.5 \mathrm{~V}$, with a 


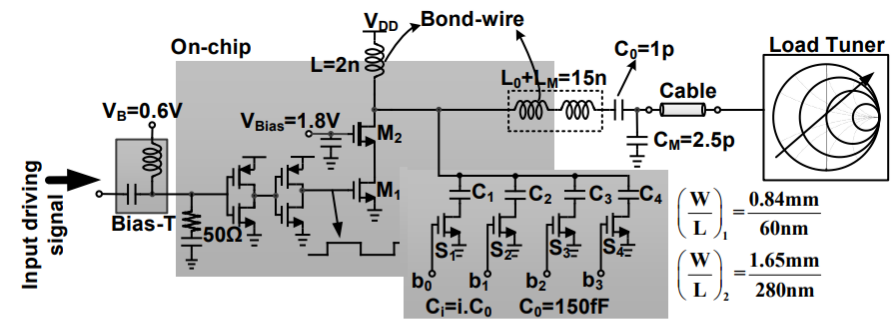

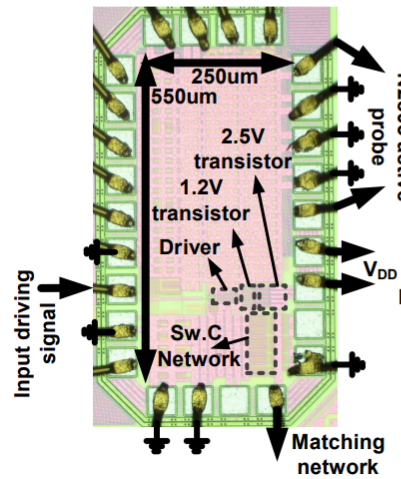

(b)

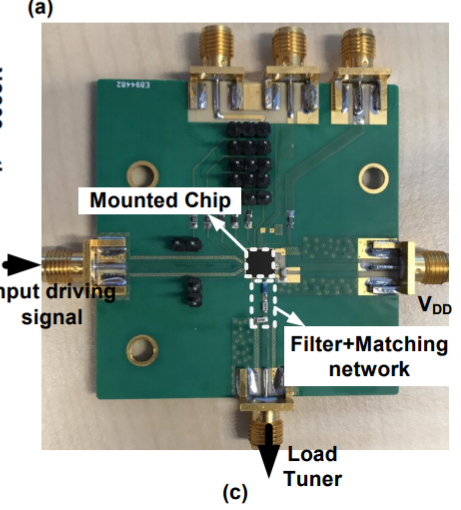

(c)
Fig. 3: (a) Schematic representation of single-ended Class-E PA with capacitor bank for tuning of the q parameter, matching network and tuner. (b) Chip microphotograph. (c) Designed PCB.

$10 \%$ technology margin). Using the K-design set elements for $q=1.4, m=0.05$ and $\alpha=\beta=0$ and for $R=25 \Omega$, $f_{0}=1.4 \mathrm{GHz}$, yields $L=2 \mathrm{nH}, C=3.3 \mathrm{pF}$ and $X \approx 0 j \Omega$. Two parallel bond-wires implement $L$ and the tank capacitor $C$ was implemented as the sum of the drain-bulk and gate-drain parasitic capacitances of the cascode transistor. Two cascaded inverters were used as the switch driver.

The variable switch node capacitor is implemented as a SCB with 4 control bits, with a total $C_{\text {bank }}=1.5 \mathrm{pF}$. The switches are implemented by $2.5 \mathrm{~V}$ thick oxide transistors and dimensioned such that the voltage excursions across them do not exceed $3 \mathrm{~V}$. The extra capacitive loading at the switching node, introduced by the $\mathrm{SCB}$, is compensated by reducing the main switch size, lowering efficiency under nominal load conditions by less than $2 \%$.

A Maury load tuner was used to implement the load-pulling for VSWRs ranging from 1:1 to over 10:1 as shown in Fig 3(a). An off-chip matching network was used to step the $50 \Omega$ load down to $R=25 \Omega$. The chip micro-photograph and the designed PCB are shown in Fig 3(b) and (c), respectively. For all the measurements in this manuscript, the power loss in the matching network is included in our power and efficiency numbers.

\section{A. Measurement results with fixed $q$ parameter}

Under nominal load conditions (VSWR=1:1) with no tuning, at $V_{D D}=1.2 \mathrm{~V}$, at $1.4 \mathrm{GHz}$, the PA provides $17.5 \mathrm{dBm}$ into a $50 \Omega$ load at $67 \%$ efficiency.

The PA performance and reliability related parameters were also measured under load-mismatch for a fixed $q=1.4$ (i.e. all the switches in the SCB in the off-state). For reliability and reproducibility reasons, the supply voltage, $V_{D D}$, was varied to keep the PA peak switch voltage below or equal to the peak switch voltage for $Z=R$ to prevent PA breakdown. To equalize the dataset, the data for these loads was scaled to $V_{D D}=1.2 \mathrm{~V}$ during post-processing, assuming $P_{\text {out }} \propto V_{D D}^{2}$, which is valid according to the theory [7]. Additionally, the output power and average switch current data are normalized to the nominal case $(\Gamma=0)$.

Due to the effect of the cables and matching network, the measured contours are rotated with respected to the theory. For clarity sake, the data was counter-rotated during processing to align it to the theoretical contours. The processed measurement results for the PA are shown in Fig. 4, where the center of

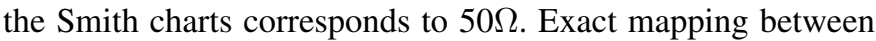
the theory and the measurements is not the focus of this manuscript. The difference can be explained by second order effects that were not taken into account in our simplified theoretical model. Despite this, the measurement data shows a trend that strongly resembles the theoretical results.

The maximum switch voltage and maximum average switch current under heavy load mismatch can go up to $6.5 \mathrm{~V}$ $\left(5.4 \times V_{D D}\right)$ and $200 \mathrm{~mA}$, respectively, compared to, $V_{c, \text { nom }}$ $=4 \mathrm{~V}\left(3.3 \times V_{D D}\right)$ and $80 \mathrm{~mA}$ under nominal load conditions when $\mathrm{q}$ is fixed. The extreme switch voltage of $6.5 \mathrm{~V}$, toward the upper left of the Smith chart, for a VSWR of 19:1, can instantly break down the PA switch. The border between the safe (peak voltage $\leq 4.6 \mathrm{~V}$ ) and non-safe areas is shown as a solid red line. Additionally, the average current of the switch can reach levels up to 2.5 times higher than under nominal condition, which can gradually degrade switch performance.

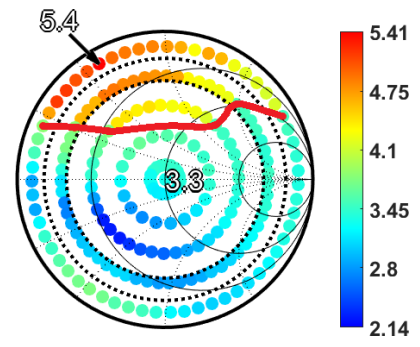

(a) Normalized peak switch

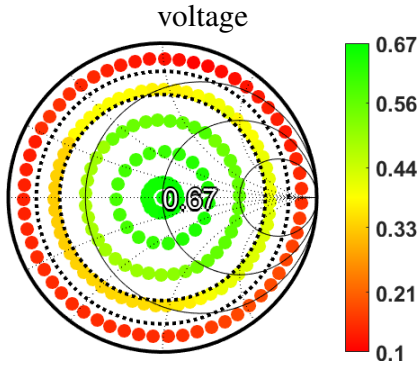

(c) Efficiency

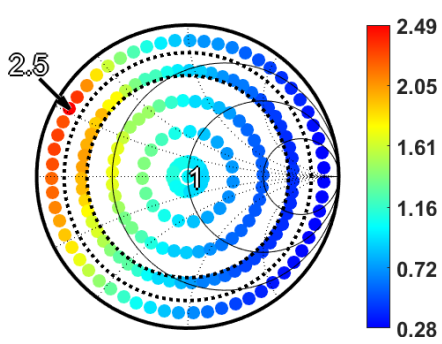

(b) Normalized average switch current

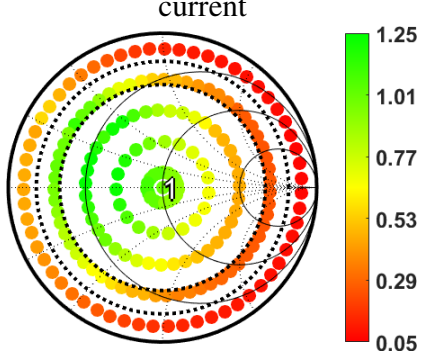

(d) Normalized output power
Fig. 4: Measured load-pull color gradients with self-heal loop deactivated

\section{B. Self-healing Class-E PA}

For self-healing, the $q$ parameter is tuned automatically to extend the safe operating region toward the upper left, with 


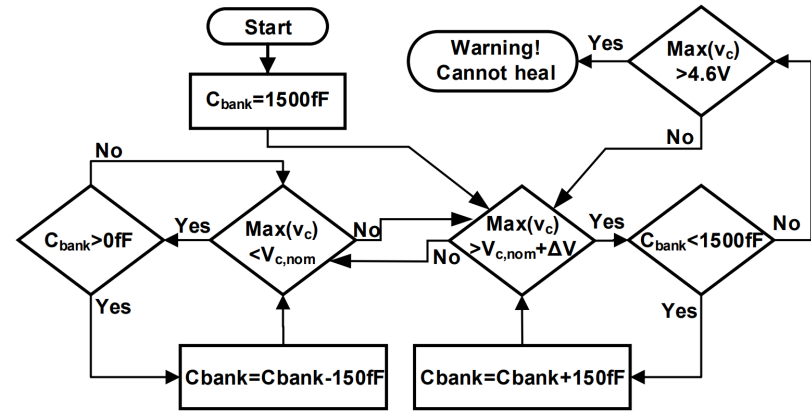

Fig. 5: Flow diagram of the self-healing algorithm.

respect to the nominal case $(q=1.4)$. A demonstrator to experimentally validate this is presented below.

For every applied load setting, the waveform at the switch node was measured using an Agilent N2803A active probe connected to an 80GSa/s Agilent oscilloscope. A simple algorithm, shown in Fig. 5, controls the capacitor bank state. A dead zone of $\Delta V=0.2 \mathrm{~V}$ was added to decrease the noise sensitivity of the self-healing algorithm.

The algorithm checks the maximum switch voltage, $V_{c, \max }$. If $V_{c, \max }<V_{c, \text { nom }}$, the self-healing loop remains idle. For voltages $V_{c, \max }>V_{c, \text { nom }}+\Delta V=4.2 \mathrm{~V}$, the self-healing loop incrementally increases the SCB state until the peak switch voltage return to a safe value. When the load returns to the nominal condition, the SCB returns to the idle state with all capacitors disconnected. If the SCB is at the maximum state and the peak switch voltage still exceeds the breakdown limit, a warning is given. Fig. 6 shows the processed measurement results for the automatic self-healing PA system.

Power and efficiency are hardly affected by the self-healing loop. The worst case deviations in efficiency and output power are an $11 \%$ relative reduction (from $56 \%$ to $50 \%$ ) and a $1.1 \mathrm{~dB}$ reduction (from $79 \mathrm{~mW}$ to $61 \mathrm{~mW}$ ), respectively. The minor efficiency reduction is due to second order effects such as extra loss introduced by the switches in the SCB.

The safe region for instant switch breakdown $\left(\leq 3.8 \times V_{D D}\right.$ , 4.6V) is considerably improved extending over the entire measured VSWR range of 19:1. More than 1.4 times reduction in maximum switch voltage can be seen toward the left upper side of the Smith chart. The average switch current safe region is also improved for high VSWRs up to 19:1. However, still 2.3 times more average current passes through the switch for extreme load mismatch. Therefore, in the design of the PA a safety factor 2.5 was assumed to ensure the PA can handle the high currents present under heavy load mismatch conditions.

\section{CONCLUSIONS}

The reliability of Class-E PAs can be heavily compromised in the presence of load-mismatch. This work introduces, to the authors' best knowledge, the first self protective/healing ClassE PAs. It was shown that decreasing the relative resonance frequency ( $q$ parameter) by employing a digitally variable added switch capacitance, and using a simple algorithm, protected the PA against over-voltage over a wide range of load mismatches

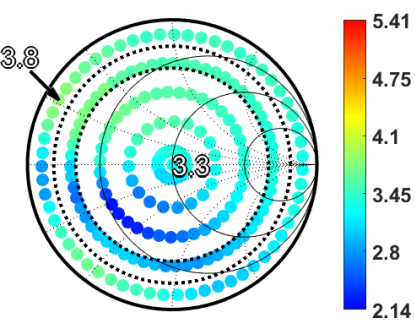

(a) Normalized peak switch voltage

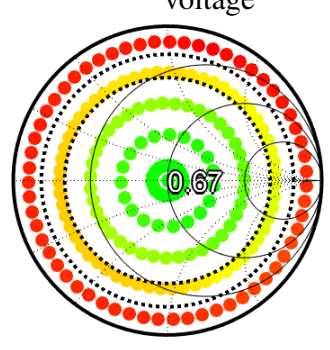

(c) Efficiency

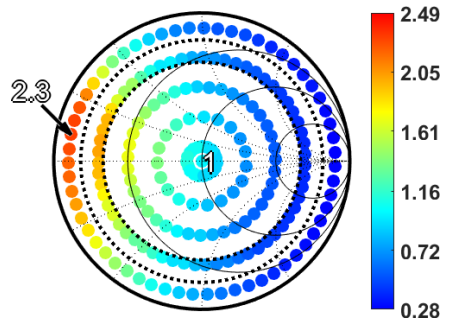

(b) Normalized average switch current

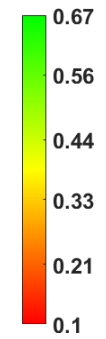

0.1

(d) Normalized output power
Fig. 6: Measured load-pull color gradients with self-heal loop activated

with VSWRs as high as 19:1. In so doing, efficiency and output power were not considerably affected. This self-healing Class-E PA in standard $65 \mathrm{~nm}$ CMOS technology provides good performance and significantly increased immunity to load variation related degradation, allowing practical application in systems subject to strong antenna load mismatch with strongly reduced risk to reliability.

A future work will include a receiver-like structure to replace the oscilloscope in the self-protective/healing system.

\section{REFERENCES}

[1] S. Cripps, RF Power Amplifiers for Wireless Communications. Artech House, 1999.

[2] F.H. Raab, Idealized operation of the class-E tuned power amplifier, IEEE TCAS, vol.24, no.12, pp. 725-735, 1977.

[3] K. Boyle and M. Leitner, Mobile phone antenna impedance variations with realusers and phantoms, Proc. iWAT, pp. 420-423, Hong Kong, 2011.

[4] I. Aoki et al.," "A Fully-Integrated Quad-Band GSM/GPRS CMOS Power Amplifier," in IEEE JSSC, vol. 43, no. 12, pp. 2747-2758, 2008.

[5] C. Kim, Electromigration in thin films and electronic devices : Materials and reliability. Oxford: Woodhead Pub.

[6] M. Acar (2011). Power amplifiers in CMOS technology : a contribution to power amplifier theory and techniques, Doctoral dissertatio, University of Twente, Enschede, The Netherlands.

[7] A. Ghahremani et al., "Outphasing Class-E Power Amplifiers: From Theory to Back-Off Efficiency Improvement," in IEEE JSSC, vol. 53, no. 5, pp. 1374-1386, 2018.

[8] S. M. Bowers et al., "Integrated Self-Healing for mm-Wave Power Amplifiers," IEEE T-MTT, vol. 61, no. 3, pp. 1301-1315, 2013.

[9] A. Scuderi et al., "VSWR-protected silicon bipolar power amplifier with smooth power control slope," 2004 IEEE ISSCC, pp. 194-522 Vol.1, San Francisco, CA, 2004.

[10] W. Karoui and T. Parra, "A protection circuit for HBT RF Power Amplifier under load mismatch conditions," Joint 6th IEEE NWCS and TAISA, Montreal, QC, pp. 241-244, 2008.

[11] N. Zimmermann et al., "An over-voltage protection circuit for CMOS power amplifiers," 15th IEEE ICECS, St. Julien's, pp. 161-164, 2008.

[12] C. Wei et al., "A novel VSWR-protected and controllable CMOS class E power amplifier for Bluetooth applications," 4th IEEE CIEA, Xi' an, pp. 420-424, 2009. 